\title{
A propósito de los nuevos anticoagulantes. Algunos datos sobre la prevalencia del riesgo de sangrado en población anticoagulada de una zona básica de salud rural.
}

\section{Sr. Editor:}

La morbimortalidad atribuible a procesos trombóticos y embólicos, en relación con el tromboembolismo venoso (TEV), la trombosis venosa profunda (TVP), el tromboembolismo pulmonar (TEP) y la fibrilación auricular (FA), es un problema primordial de salud en los países desarrollados y en vías de desarrollo.

Hasta hace una década, para su tratamiento contábamos con heparinas no fraccionadas o de bajo peso molecular, el más moderno fundaparinux y los anticoagulantes orales, inhibidores de la vitamina $\mathrm{K}$, acenocumarol y warfarina. Pero en los últimos años estamos presenciando la introducción en clínica de nuevos anticoagulantes orales -dabigatran, rivaroxaban y apixaban- que no precisan monitorización como los anteriores y han demostrado su eficacia en la profilaxis de TEV, el tratamiento de la TVP y del TEP, y la profilaxis del cardioembolismo debido a fibrilación auricular no valvular (FANV).

Aunque presentan mayor seguridad clínica por la menor incidencia de hemorragia intracraneal, su uso no está libre de problemas. El dabigatran, un inhibidor de la trombina, tiene alertas por sangrados fatales, sobre todo en pacientes mayores, y está contraindicado en insuficiencia renal grave y, según la Agencia Española de Medicamentos y Productos Sanitarios (AEMPS), también en la fibrilación auricular valvular. Igualmente, se ha relacionado con un aumento del riesgo de infarto de miocardio y otros síndromes coronarios agudos, y presenta numerosas interacciones (AAS, clopidogrel, AINES, inhibidores de la bomba de protones, etc.)

Respecto al rivaroxaban, un inhibidor directo del factor Xa altamente selectivo, está contraindicado en insuficiencia hepática y renal grave (aclaramiento de creatinina $<15 \mathrm{ml} / \mathrm{min}$ ), y se han descrito interacciones con ketoconazol, variconazol, voriconazol y ritonavir, carbamazepina, fenitoína, rifampicina y hierba de San Juan.

A la vista de lo anterior, la Sociedad Española de Medicina de Urgencias y Emergencias y la de Cardiología recomiendan anticoagular sólo a pacientes en los que exista un riesgo trombótico significativo (CHADS2, CHA2DS2-VASc) y mantenerlo de manera crónica ${ }^{1}$. La Sociedad Española de Medicina de Familia y Comunitaria (SEMFYC) coincide en la indicación de anticoagulación en pacientes con riesgo significativo de trombosis si éste supera al de sangrado (HAS-BLED) y sugiere que el dabi- gatran podría ser una alternativa en pacientes con FANV y un riesgo significativo de trombosis en los que el control mediante el INR fuera muy irregular (más del $40 \%$ de los controles fuera de rango), en cuyo caso se debería monitorizar la función renal y la aparición de efectos adversos, sobre todo sangrados $^{2}$. Por su parte, la AEMPS recomienda el uso de los nuevos anticoagulantes en los siguientes casos $^{3}$ :

- Pacientes con antecedentes de hemorragia intracraneal (HIC) en los que se valore que los beneficios de la anticoagulación superan el riesgo hemorrágico.

- Pacientes con antecedentes de ictus que presenten criterios clínicos o de neuro-imagen de alto riesgo de HIC (en los cuales los nuevos anticoagulantes podrían ser la alternativa ideal), definido como la combinación de HAS-BLED $\geq 3$ y al menos una de las siguientes: leucoaraiosis grados II-IV y/o microsangrados corticales múltiples.

En este contexto, queremos aportar algunos datos sobre la situación de nuestros pacientes anticoagulados, con sus circunstancias reales (ancianos, pluripatología, polimedicación, medio rural, lejanía a hospital, escasez de recursos, etc.), para valorar cuántos pacientes anticoagulados tenemos realmente con riesgo de sangrado significativo y en cuántos sería adecuado el cambio de los clásicos inhibidores de vitamina $\mathrm{K}$ por los nuevos anticoagulantes. Los datos pertenecen a tres de nuestros cupos, rurales, que comprenden 1.769 pacientes repartidos en siete núcleos de población de nuestra zona de salud. En total identificamos 67 pacientes anticoagulados $(3,7 \%)$, a los que calculamos el riesgo cardioembolígeno, mediante CHA2DS2VASc, y el riesgo hemorrágico, por HAS-BLED.

Se detectó riesgo de sangrado significativo (HAS$B L E D \geq 3$ ) en 51 casos $(76,1 \%)$. Fue mayor el riesgo de trombosis que el de sangrado en 53 de los 67 $(79,1 \%)$, similar en $12(17,9 \%)$ y sólo en 2 pacientes $(3,0 \%)$, superó el riesgo de hemorragia al de trombosis. Vemos que parece existir una elevada prevalencia de pacientes anticoagulados en el medio rural con riesgo de sangrado significativo. Esto es debido tanto al progresivo envejecimiento como a la frecuente comorbilidad de la población que atendemos.

Decisiones tan relevantes como la adecuada elección entre un anticoagulante tradicional o un nuevo anticoagulante se deben tomar desde el contexto 
y circunstancias del paciente, de sus preferencias, de su red social y de su familia, de la comunidad a la que atendemos e, incluso, del momento actual de crisis de recursos en las que estamos ${ }^{4}$. Señala el Centro Andaluz de Documentación e Información de Medicamentos en su evaluación del dabigatran5: "Considerando el coste/tratamiento año y estimando unos costes asociados a la monitorización del INR de 320-750 euros/paciente/año, el coste incremental de dabigatran respecto la terapia de referencia (acenocumarol o warfarina) oscilaría entre 436 y 856 euros por paciente/año. Teniendo en cuenta que habría que tratar a 92 pacientes durante dos años con dabigatran 150 cada 12 horas en lugar de warfarina para evitar un nuevo caso de ictus o embolismo sistémico, el coste para evitar un caso adicional oscilaría entre 74.244 y 149.064 euros". Las conclusiones de este análisis, como vemos, son desmoralizadoras. Un análisis coste-utilidad, que contemplara la comodidad de no tener controles, al menos, mensuales, mediante molestos pinchazos, ahorrar desplazamientos y tiempo a sanitarios, podría arrojar unas sensaciones más favorables. Por último, el análisis coste-efectividad (condiciones reales, con efectos secundarios menores, que causan algún abandono, con sangrados mayores, algunas incluso mortales; las pérdidas de seguimiento de pacientes que antes acudían cada mes; contraindicaciones de uso en FA valvular e insuficiencia renal; monitorizaciones en pacientes ancianos, etc.), también ponderan el entusiasmo de "ahorrarnos los controles mensuales" y la supuesta seguridad de "estar siempre en rango".

La AEMPS deja claro que en todos los pacientes con alto riesgo de sangrado y datos en neuroimagen sugerentes de riesgo de sangrado intracerebral deberían utilizarse los nuevos anticoagulantes. Así pues, en nuestro caso concreto, ¿deberíamos hacer una tomografía axial computerizada al 76,1\% de nuestros pacientes?

Pensamos que, a pesar de nuestro afán por la beneficencia y de decantarnos por estas novedades, aparentemente cómodas y de fácil manejo, debemos contenernos y no tomar decisiones poblacionales simples, dados los cuestionamientos expuestos en cuanto a su seguridad (no maleficiencia) y su elevado coste (justicia o equidad).

\section{BIBLIOGRAFÍA}

1. Suero Méndez C. Los nuevos anticoagulantes orales en la Fibrilación Auricular: preguntas y respuestas para el Urgenciólogo. Emergencias. 2013;25(2):123-136.

2. Uso Racional del Medicamento. Monográfico. AMF. 2012;8(5):279-280.

3. Agencia Española del Medicamentos y Productos Sanitarios. Criterios y Recomendaciones generales para el uso de nuevos anticoagulantes orales en la prevención del ictus y la embolia sistémica en pacientes con fibrilación auricular no valvular (19 de diciembre del 2012). Disponible en: http://www.aemps.gob.es/medicamentosUsoHumano/informesPublicos/docs/criterios-anticoagulantes-orales.pdf

4. Baos Vicente V. Costes, beneficios y riesgos de los nuevos anticoagulantes. FMC. 2013;20(5):243-45.(7)

5. Centro Andaluz de Documentación e Información de Medicamentos (CADIME). Informe de evaluación de evaluación de Dabigatran (DCl). Marzo 2012. Disponible en: http:// www.cadime.es/docs/informes/CADIME_INT2012_DABIGATRAN.pdf

\section{Roel Ramírez Gonzáleza, Gabriel Sánchez Navab, José Félix Sastre Garcíab, José Juan Carbayo Garcíab.}

a Residente de tercer año de Medicina de Familia y Comunitaria

${ }^{\mathrm{b}}$ Especialista en Medicina de Familia y Comunitaria

Gerencia de Área Integrada de Talavera de la Reina (Toledo). Servicio de Salud de Castilla-La Mancha

Correo electrónico: josefelixsast@gmail.com 